\title{
The levels and trends of raised total cholesterol at national and sub-national scale in Iran from 1990 to 2016: systematic review and pooled analysis
}

\section{Parinaz Mehdipour}

Non-Communicable Diseases Research Center,Endocrinology and Metabolism Population Sciences Institute, Tehran University of Medical Sciences,Tehran,Iran

\section{Sadaf G. Sepanlou}

Digestive Diseases Research Center,Digestive Diseases Research Institute,Tehran University of Medical

Sciences, Tehran,Iran

\section{Farnam Mohebi}

Non-Communicable Diseases Research Center,Endocrinology and Metabilism Population Sciences Institute,Tehran University of Medical Sciences,Tehran,Iran

\section{Alireza Ahmadvand}

School of Clinical Sciences, Faculty of Health, Queensland University of Technology, Brisbone, Australia Niloofar Peykari

Ministry of health and Medical Education,Tehran,Iran

\section{Shirin Djalalinia}

Deputy of Research and Technology,Ministry of Health and Medical Education,Tehran,Iran

\section{Ehsan Rezaei-Darzi}

Non-Communicable Diseases Research Center, Endocrinology and Metabolism Population Sciences Institute, Tehran University of Medical Sciences, Tehran, Iran

\section{Yousef Moradi}

Pars Advanced and Minimally Invasive Medical Manners Research Center,Pars Hospita;Iran University of Medical Sciences, Tehran,Iran

\section{Rosa Haghshenas}

Non-Communicable Diseases Research Center,Endocrinology and Metabolism Population Sciences Institute,Tehran University of Medical Sciences, Tehran, Iran

\section{Shohreh Naderimagham}

Non-Communicable Diseases Research center,Endocrinology and Metabolism Population Sciences Institute,Tehran University of Medical Sciences,Tehran,Iran

\section{Hamidreza Jamshidi}

School of Medicine, Department of Pharmacology, Shahid Beheshti University of Medical Sciences, Tehran,Iran

\section{Farshad Farzadfar ( $\nabla$ f-farzadfar@tums.ac.ir )}


Non-Communicable Diseases Research Center, Endocrinology and Metabolism Population Sciences Institute, Tehran University of Medical Sciences, Tehran, Iran https://orcid.org/0000-0001-8288-4046

\section{Research article}

Keywords: Cholesterol; Hypercholesterolemia; Elevated; Raised; Prevalence; Iran; Longitudinal studies Posted Date: October 14th, 2019

DOI: https://doi.org/10.21203/rs.2.16011/v1

License: (c) (1) This work is licensed under a Creative Commons Attribution 4.0 International License. Read Full License 


\section{Abstract}

Raised serum cholesterol is a main risk factor for all-cause and cardiovascular-attributable mortality and morbidity in all nations, particularly in developing countries. Effective policy making in this regard requires detailed data to distinguish disparities and specific patterns. Thus, we aimed to estimate the level and trends of serum cholesterol level and raised total cholesterol prevalence at subnational level from 1990 to 2016 in Iran.We conducted a comprehensive systematic review, through which we detected nine national surveillances, seven large-scale population-based studies, and 88 studies published in databases. We conducted a two-stage model: an Age-Spatio-Temporal model and a Gaussian Process Regression to estimate mean total cholesterol (TC). We used crosswalk to estimate prevalence of raised total cholesterol based on estimated mean, defined as TC higher than $200 \mathrm{mg} / \mathrm{dL}$. All estimations were done in subgroups with sex, age, year, and province combinations. At national level, the number of adults with raised total cholesterol decreased from $10.5(9 \cdot 8-11 \cdot 5)$ million (5.2 million in women) in 1990 to $9.9(9.0-10 \cdot 6)$ million ( 5.4 million in women) in 2016. Age standardized prevalence of raised total cholesterol at national level has decreased from $57 \cdot 2 \%$ (95\% Cl: $53 \cdot 3-61 \cdot 1)$ to $22 \cdot 4 \%(20 \cdot 5-24 \cdot 3)$ in women and from 53.2\% (49.1 - 57.3) to $18 \cdot 0 \%(16 \cdot 4-19 \cdot 6)$ in men between 1990 and 2016. From 1990 to 2016 , age standardized mean TC in both men and women has decreased from $197 \cdot 3 \mathrm{mg} / \mathrm{dL}(186.1-$ $209 \cdot 4)$ to $168.5(160.7-176 \cdot 7)$ and from $202.5 \mathrm{mg} / \mathrm{dL}(191.2-214 \cdot 6)$ to $174.5 \mathrm{mg} / \mathrm{dL}(166 \cdot 6-182 \cdot 8)$, respectively. In both sexes, population growth had the highest contribution to increase in number of adults with high TC.Decrease in raised TC is likely the result of statin widespread use, food industry improvements, and the expanded primary health care of the country. Provided data at subnational level would help with further evidence-based and population-specific policy making to ensure the continuation of this successful experience, particularly targeting inter-gender and inter-provincial differences. Finally, the increased number of adults with TC slightly below the $200 \mathrm{mg} / \mathrm{dL}$ cutoff is threat for the healthcare that should be systematically managed.

\section{Background}

The world is witnessing a rapid transition from communicable to non-communicable diseases (NCDs)(1). The World Health Assembly developed an action plan at global level in 2013 to confront the epidemic of NCDs and to achieve a $25 \%$ reduction in risk of premature mortality from cardiovascular diseases, cancers, diabetes, and chronic respiratory diseases till 2020(2). This plan can be implemented mainly through a comprehensive primary prevention to control the metabolic risk factors of NCDs.

Raised total cholesterol is recognized as one of the main four metabolic risk factors of major NCDs $(3,4)$. There is compelling evidence on causal relationship between this risk factor and cardiovascular diseases and some cancers. Previous evidence shows an increasing trend of raised total cholesterol (TC) and the burden attributable to it across the world and even in low income developing countries (5). However, due to data scarcity, difficulties of measuring total cholesterol, and various definitions for high TC, the evidence on levels and trends of this risk factor are not consistent, not adequate, and not comparable 
across regions and nations. Collecting high quality data at global, regional, national, and even subnational levels is thus a crucial step for evidence-based policy making in low resource settings.

In the current study, we aimed to quantify the levels and trends of mean total cholesterol, to estimate the distribution and prevalence and also the number of adults with raised total cholesterol at national and sub-national levels in Iran from 1990 to 2016.

\section{Methods}

In the current study, we estimated the mean total cholesterol and prevalence of high TC by sex and age across all provinces of Iran from 1990 to 2016. TC equal or higher than $170 \mathrm{mg} / \mathrm{dL}$ and equal or higher than $200 \mathrm{mg} / \mathrm{dL}$ was considered elevated TC (pre-hypercholesterolemia) and raised TC (hypercholesteremia), respectively (6). Both sexes, 13 age groups from 25 to over 85 years (5-year categories), 31 provinces, and 27 years from 1990 to 2016 are covered in our analyses.

We initiated our study with a comprehensive systematic review on available evidence. We identified nine national surveys in Iran, constituting two iterations of national health surveys (NHS) in 1991 and 2000, seven iterations of NCD Risk Factors Surveillance Surveys in (STEPwise), and seven population-based studies. We obtained individual-level data of national surveys and tabulated data from the populationbased studies. We extracted the data of 68 published studies that we found through the systematic review and we finally extracted 88 data points from studies. The details of systematic review are presented in the appendix (page 3).

In order to estimate the mean total cholesterol by sex, age, province, and year, we used a Generalized Linear Mixed Model (GLMM), with variables including wealth index, years of schooling, and urbanization as fixed-effects covariates and province with random effects for the model (7). We ran an Age-SpatioTemporal model on the residuals extracted from GLMM, in which we borrowed strengths over age groups, provinces, and years by smoothing the residuals (8). Finally, we used the output of previous models as a prior for Gaussian Process Regression (GPR). The details are described in appendix (page 7) $(7,9)$.

In order to estimate the prevalence of elevated and raised TC, we used a cross-walk method to regress prevalence over mean TC, extracted from the GPR model.

We conducted a sensitivity analysis to examine the validity of our estimations (10). We randomly selected $10 \%$ of our original data points and dropped them. We redid all the models on the remaining $90 \%$. We compared the in-sample validity using the following four metrics: Root Mean Square Error (RMSE), Root Median Square Error, Median Relative Error, and Mean Relative Error of prediction models. In the next stage we examined the proportion of the originally dropped $10 \%$ data points that were within the $95 \%$ uncertainty Interval of our $90 \%$ withheld data.

We calculated the percent change in number of adults with elevated TC. We decomposed the contribution of decreased age-standardized prevalence, population growth, and change in age structure to the percent 
change in number of adults with elevated TC.

\section{Role of funding source}

The funder of the study had no role in study design, data collection, analysis, interpretation, and drafting the report. FF, as the corresponding author, had full access to the data in the study and had final responsibility for the decision to submit for publication.

\section{Results}

We conducted a comprehensive systematic review and pooled 3,505 data points on over 500,000 adults, aged 25 to over 85 years, recruited in national and provincial population-based surveys in Iran as well as published studies from 1990 to 2016 . The number of data sources for each province and year is demonstrated in the heat map in appendix (Supplementary Figure 1).

Nationally, the number of adults with total cholesterol over $200 \mathrm{mg} / \mathrm{dL}$ decreased from $10.5(9.8-11.5)$ million in 1990 to $9.9(9.0-10 \cdot 6)$ million in 2016. In the meantime, the number of adults with elevated TC, or TC over $170 \mathrm{mg} / \mathrm{dL}$, increased from $15.6(15 \cdot 0$ - 16.1) million (7.6 million in women) in 1990 to $22 \cdot 9(21 \cdot 6-24 \cdot 2)$ million (12.0 million in women) in 2016.

Age standardized prevalence of raised total cholesterol at national level has decreased from $57 \cdot 2 \%$ (95\% Cl: $53.3-61 \cdot 1)$ to $22.4 \%(20.5-24.3)$ in women and from $53.2 \%(49.1-57 \cdot 3)$ to $18.0 \%(16 \cdot 4-19.6)$ in men between 1990 and 2016 (Figures 1). Age standardized prevalence of elevated TC at national level has decreased from $82 \cdot 4 \%(79 \cdot 8-84 \cdot 8)$ to $49 \cdot 5 \%(46 \cdot 8-24 \cdot 4)$ in women and from $79 \cdot 7 \%(76 \cdot 7-82 \cdot 3)$ to $43 \cdot 7 \%(41 \cdot 0-46 \cdot 5)$ in men between 1990 and 2016 (Figure 1 and supplementary Figure 2).

From 1990 to 2016, age standardized mean TC in women has decreased from $202.5 \mathrm{mg} / \mathrm{dL}(191 \cdot 2-$ $214 \cdot 6)$ to $174.5 \mathrm{mg} / \mathrm{dL}(166 \cdot 6-182 \cdot 8)$. In men mean TC decreased from $197 \cdot 3 \mathrm{mg} / \mathrm{dL}(186 \cdot 1-209 \cdot 4)$ to $168 \cdot 5(160 \cdot 7-176 \cdot 7)$ (Figure 1).

The percent change in age-standardized prevalence of elevated TC from 1990 to 2016 was $-40 \%$ ( -45 to -35) in women and $-45 \%$ (-50 to -39) in men (Supplementary Table 1). The percent change in agestandardized prevalence of raised TC from 1990 to 2016 by $-61 \%(-66 \%$ to $-54 \%)$ in women and $-66 \%(-71$ to -60) in men (Supplementary Figure 3). The prevalence of elevated TC has decreased in all provinces and decreased by more than half in a few provinces in both sexes from 1990 to 2016 . Similarly, the prevalence of raised TC has decreased by more than half in almost all provinces in both sexes from 1990 to 2016, except for one province in men and four provinces in women (Figure 2 and 3).

Mean TC, and prevalence of both elevated and raised TC increased in adults from 25 to 55 years of age and then showed a steady trend till the oldest age group of 85 and over in all years from 1990 to 2016 (supplementary figure 4). The age pattern of elevated TC prevalence similarly increased between 25 and 55 age groups and then it was steady in all provinces in 2016. Yet, there is an evident convergence in 
elevated TC prevalence below 40 years of age and a clear divergence in older age groups (Supplementary Figure 5).

Across provinces in 1990, age standardized prevalence for TC equal or greater than 200 in women ranged from $34 \cdot 0 \%(28 \cdot 7 \%-39 \cdot 6 \%)$ to $75 \cdot 5 \%(70 \cdot 0 \%-78 \cdot 4 \%)$, and in 2016 the range was as low as $17 \cdot 8 \%$ $(16.0 \%-19 \cdot 7 \%)$ to as high as $27.5 \%(24.9 \%-30 \cdot 2 \%)$. In men, the age standardized prevalence in 1990 ranged from $33 \cdot 3 \%(28 \cdot 6 \%-38 \cdot 4 \%)$ to $74 \cdot 9 \%(71 \cdot 4 \%-78 \cdot 2 \%)$ and in 2016 , from $13 \cdot 1 \%(11 \cdot 8 \%-14 \cdot 6 \%)$ to $23 \cdot 8 \%(21 \cdot 4 \%-26 \cdot 4 \%)$ (Figure 2, Supplementary Figure 2). These measures for elevated TC are presented in supplementary figures 2 and 3.

In 1990, there were no provinces with mean TC less than $170 \mathrm{mg} / \mathrm{dL}$ in women and in 21 provinces, the mean TC was over $200 \mathrm{mg} / \mathrm{dL}$, while in 2016 there was no province with mean TC over $200 \mathrm{mg} / \mathrm{dL}$ and in five provinces the mean TC was even less than $170 \mathrm{mg} / \mathrm{dL}$. In men, in 1990, there were no provinces with TC less than $170 \mathrm{mg} / \mathrm{dL}$, while in 2016 the mean TC was less than $200 \mathrm{mg} / \mathrm{dL}$ in all provinces and even less than $170 \mathrm{mg} / \mathrm{dL}$ in 22 provinces, and between 170 to $199 \mathrm{mg} / \mathrm{dL}$ in the remaining 9 provinces (Supplementary Figure 2).

In terms of raised total cholesterol prevalence, 18 provinces had decreasing percent change greater than $60 \%$ in women. While in men, 25 provinces had decreasing percent change greater than $60 \%$. It shows that decreasing progress was more rapid in men compared to women (Supplementary Figure 6).

Figure 4 demonstrates the shift in the distribution of TC, from 1990 to 2016 . This pattern shows that the mean of the entire distribution has been shifted to left; however, the standard deviation of distribution has also decreased. The decrease was more prominent in men compared to women.

In women across all provinces, the mean age standardized TC in 2016 ranged from $166 \cdot 4 \mathrm{mg} / \mathrm{dL}$ (154.1 - 179.6) to $182.3 \mathrm{mg} / \mathrm{dL}(171.4-193.8)$. In men, respective measures for TC were as low as 159.0 $\mathrm{mg} / \mathrm{dL}(150 \cdot 1-168 \cdot 2)$ to as high as $178.2 \mathrm{mg} / \mathrm{dL}(168 \cdot 0-189 \cdot 0)$ in 2016 . (Supplementary Figures 1 and 2).

In terms of the trend of mean TC from 1990 to 2016, the percent decrease ranged from $-23 \cdot 6 \%(-36 \cdot 3-$ $-8 \cdot 8)$ to $-5 \%(-20 \cdot 1-13 \cdot 1)$ in women. The equivalent results in men were $-24 \cdot 8 \%(-33 \cdot 5--14 \cdot 9)$ to $-0 \cdot 6 \%$ $(-15 \cdot 3-16 \cdot 3)$ (Supplementary Figure 2).

For sensitivity analysis, we calculated the proportion of data points in our masked data set that were located in the $95 \%$ Uncertainty Interval of our 90 percent withheld data. For TC, $82 \cdot 7$ percent of $10 \%$ withheld data hold in $90 \%$ prediction of model and its uncertainty.

\section{Discussion}

The results of our study showed a consistently decreasing trend in mean TC and prevalence of raised total cholesterol across both sexes, all age groups, and all provinces in Iran from 1990 to 2016. While the number of adults with raised TC decreased from 1990 to 2016, we observed an increase in the number of 
adults with elevated TC in this period. This increase was more prominent in women; $57 \cdot 9 \%$ in women, and $36.2 \%$ in men. At subnational level and among both men and women, the prevalence of both elevated and raised TC has decreased, however, the decrease was bigger for elevated TC. We also observed a shift in distribution of mean TC to left across provinces with a similar decrease in standard deviation, more dominant in men.

Generally, our results are compatible with estimates made by Farzadfar et al on mean TC and its trend from 1980 to $2008(5)$. They reported that in $2005,16,000$ deaths $(14,000$ to 18,000$)$ in women and 18,000 deaths $(16,000$ to 20,000$)$ in men were attributable to high TC (11). The Global Burden of Disease Study showed that in $1990,11,100$ deaths $(8,300$ to 14,300$)$ in women were attributable to raised TC while the respective figures were $19,400(13,700$ to 25,800$)$ in 2016 . Respective figures for men were 15,000 $(11,200$ to 19,600$)$ deaths in 1990 and 27,500 (19,600 to 37,000) deaths in 2016(4). In comparing our observations to international trends, the observed decreased in the mean TC and also the prevalence of raised TC in our study was among the highest in previously reported time trends (12-15). This finding shows the probable contribution of effective treatment in decreasing high levels of TC, while there are probably other determinants that have further decreased lower levels of TC. It is mainly prominent when we consider the observation that most of the adults with TC higher than $200 \mathrm{mg} / \mathrm{dL}$ has moved to the group with TC 170 to $200 \mathrm{mg} / \mathrm{dL}$. It supports the dual impacts of primary and secondary prevention on decreasing TC among Iranians since 1990. Improvement of food industry has had a certain impact. In early 2000s, a transition happened in the food industry of Iran in which, solid hydrogenated and animal fat was largely replaced by liquid vegetable oil with lower trans fatty acids. Based on a report released by the Iran Ministry of Health and Medical Education, the use of solid hydrogenated oil has been replaced by liquid vegetable oil in Iran since 1990s. This transition can be the main driver of the shift in distribution of TC to left and denotes the success of the health system in improving food industry and implementing public education. Moreover, during the past decade, the prescription of statins in both primary and secondary health care settings has become routine based on most recent guidelines for the prevention of cardiovascular diseases, which are the most important causes of morbidity and mortality attributable to high TC. An ecologic study demonstrated the negative correlation between statin sales and cholesterol level among Iranian population from 2005 to 2011(16). Finally, the differential pattern of mean and high TC in men and women. This may have biologic plausibility as well as other co-existing distal life style and cardio-metabolic risk factors in women such as overweight and obesity, diet, and physical inactivity(17-19).

Our study has several strengths. It is the first comprehensive systematic review on levels and trends of high TC at subnational scale in Iran using advanced statistical methods to overcome the data scarcity in Iran. We investigated all the available data in this regard to report the most comprehensive statistics. We also reported the measures in different subgroups to enlighten the disparities and differences, applicable for further policy making. Yet, our study has certain limitations. We had to overcome data scarcity by statistical methodology, which increased the uncertainty of our estimates. For estimating the prevalence of elevated and raised TC, we used crosswalk on our estimates for mean TC. To make estimates by 5year age groups among adults older than 65 years, we split age groups based on the data of the last 
iteration of National NCD Risk Factor Surveillance in 2016 and applied it to previous years. And finally, and most importantly, we could not differentiate Low Density Lipoprotein from High Density Lipoproteins, which imposes bias on our estimates for burden attributable to unfavorable cholesterol.

Our results are of a great importance to policy makers as follows; 1 ) the success in decreasing the prevalence of raised TC in Iranian population validates the effectiveness of previously implemented policies. Even though in the healthcare network in Iran statins are available, there is no service package specifically designed for primary and secondary prevention of high serum cholesterol and the capacity of the health system for tackling NCDs and their metabolic risk factors is inadequate(20,21). Moreover, the combination of inter-sectoral collaboration between the health systems, food industry, health education, and all organizations that are somehow involved in health and well-being of Iranians can guarantee the continuation of decreasing trend of TC in Iran. However, 2) the increased number of adults with elevated TC shows a potential threat to the health system and it shows that there are many people, that if not efficiently managed or prevented, would have raised TC anytime soon. The establishment of the Iranian Non-Communicable Diseases Committee (INCDC) is the first concerted effort to design a comprehensive service package specific to metabolic risk factors of NCDs and their integration into the health system of Iran(22). Nevertheless, the implementation and effectiveness are not evaluated yet. 3) The observed disparities among two genders necessitate further investigation of the underlying reasons while enlightens the importance of population-specific policies; what worked for men, might not work for women. And finally, 4) the different statistics of different provinces is a call for subnational designed and implemented programs to be more efficient and effective.

In conclusion, the trends of TC are improving in Iran across all provinces, both sexes, and all age groups. To continue the current success, policy makers require to maintain the previously implemented effective policies. Moreover, the success should not be taken for granted as there are a considerable number of people just below the cut point of raised cholesterol. Additionally, different provinces and different genders require different approaches that should be addressed in national programs and plans.

\section{Abbreviations}

TC: Total Cholesterol

GBD: Global Burden of Disease

GLMM: Generalized Linear Mixed Model

GPR: Gaussian Process Regression

NCD: Non-Communicable Disease

NHS: National Health Survey

RSME: Root Mean Square Error 
SDG: Sustainable Development Goals

\section{Declarations}

\section{Ethics approval and consent to participate}

Not applicable

\section{Consent for publication}

Not applicable

\section{Availability of data and materials}

The datasets used and/or analysed during the current study are available from the corresponding author on reasonable request.

\section{Declaration of Interests}

We declare no competing interests.

\section{Contributors}

FF designed and supervised the study and approved of the final version of the manuscript.

PM did entire data preparation, statistical analysis, prepared results, wrote the manuscript, edited the content of manuscript, and approved of the final version.

SGS did the comprehensive systematic review, wrote the manuscript, edited the content of manuscript, and discussed the results.

AA did the comprehensive systematic review and did entire data preparation.

FM did the comprehensive systematic review, prepared results of systematic review, edited the content of manuscript, discussed the results, and approved of the final version.

SD did data collection through contacting principal investigators of population-based studies.

NP did data collection through contacting principal investigators of population-based studies.

ER did the original data preparation and statistical analysis.

$\mathrm{RH}$ did administrative processes.

HJ supervised administrative process.

The rest of the co-authors were collaborators in our team. 


\section{Acknowledgements}

The authors would like to express their appreciation for partnership of deputy for public health and deputy for research and technology of the Ministry of Health and Medical Education, Islamic Republic of Iran's, National Institute for Health Research, and many scholars and experts in related fields. We would also like to express thanks to all of participants and scientific and executive partners of medical sciences universities, who made this experience.

\section{References}

1. Holeczek H. Renewables: Global status report 2014. Galvanotechnik. 2014;

2. WHO. Global action plan for the prevention and control of noncommunicable diseases 2013-2020. World Heal Organ. 2013;

3. Forouzanfar MH, Alexander L, Bachman VF, Biryukov S, Brauer M, Casey D, et al. Global, regional, and national comparative risk assessment of 79 behavioural, environmental and occupational, and metabolic risks or clusters of risks in 188 countries, 1990-2013: A systematic analysis for the Global Burden of Disease Study 2013. Lancet. 2015;

4. Gakidou E, Afshin A, Abajobir AA, Abate KH, Abbafati C, Abbas KM, et al. Global, regional, and national comparative risk assessment of 84 behavioural, environmental and occupational, and metabolic risks or clusters of risks, 1990-2016: A systematic analysis for the Global Burden of Disease Study 2016. Lancet. 2017;

5. Farzadfar F, Finucane MM, Danaei G, Pelizzari PM, Cowan MJ, Paciorek CJ, et al. National, regional, and global trends in serum total cholesterol since 1980: Systematic analysis of health examination surveys and epidemiological studies with 321 country-years and 3.0 million participants. Lancet. 2011;

6. Smith SC, Allen J, Blair SN, Bonow RO, Brass LM, Fonarow GC, et al. AHA/ACC guidelines for secondary prevention for patients with coronary and other atherosclerotic vascular disease: 2006 Update - Endorsed by the National Heart, Lung, and Blood Institute. Circulation. 2006.

7. Mohammadi Y, Parsaeian M, Mehdipour P, Khosravi A, Larijani B, Sheidaei A, et al. Measuring Iran's success in achieving Millennium Development Goal 4: a systematic analysis of under-5 mortality at national and subnational levels from 1990 to 2015. Lancet Glob Heal. 2017;5(5).

8. Foreman KJ, Lozano R, Lopez AD, Murray CJL. Modeling causes of death: An integrated approach using CODEm. Popul Health Metr. 2012;

9. Mehdipour P, Navidi I, Parsaeian M, Mohammadi Y, Lakeh MM, Darzi ER, et al. Application of gaussian process regression (GPR) in estimating under-five mortality levels and trends in Iran 19902013, study protocol. Arch Iran Med. 2014;17(3). 
10. Rajaratnam JK, Marcus JR, Flaxman AD, Wang H, Levin-Rector A, Dwyer L, et al. Neonatal, postneonatal, childhood, and under-5 mortality for 187 countries, 1970-2010: a systematic analysis of progress towards Millennium Development Goal 4. Lancet. 2010;

11. Farzadfar F, Danaei G, Namdaritabar H, Rajaratnam JK, Marcus JR, Khosravi A, et al. National and subnational mortality effects of metabolic risk factors and smoking in Iran: A comparative risk assessment. Popul Health Metr. 2011;

12. Eliasson M, Janlert U, Jansson JH, Stegmayr B. Time trends in population cholesterol levels 19862004: Influence of lipid-lowering drugs, obesity, smoking and educational level. The northern Sweden MONICA study. J Intern Med. 2006;

13. Ford ES, Capewell S. Trends in Total and Low-Density Lipoprotein Cholesterol among U.S. Adults: Contributions of Changes in Dietary Fat Intake and Use of Cholesterol-Lowering Medications. PLoS One. 2013;

14. Arnett DK, Jacobs DR, Luepker R V., Blackburn H, Armstrong C, Claas SA. Twenty-year trends in serum cholesterol, hypercholesterolemia, and cholesterol medication use: The Minnesota Heart Survey, 1980-1982 to 2000-2002. Circulation. 2005;

15. Borodulin K, Vartiainen E, Peltonen M, Jousilahti P, Juolevi A, Laatikainen T, et al. Forty-year trends in cardiovascular risk factors in Finland. Eur J Public Health. 2015;

16. Ahmadvand A, Farzadfar F, Jamshidi HR, Mohammadi N, Holakouie-Naieni K. Using drug sales data to evaluate the epidemiology of cardiometabolic risk factors and their inequality: An ecological study on atorvastatin and total cholesterol in Iran. Med J Islam Repub Iran. 2015;

17. Afshin A, Micha R, Khatibzadeh S, Fahimi S, Shi P, Powles J, et al. The impact of dietary habits and metabolic risk factors on cardiovascular and diabetes mortality in countries of the Middle East and North Africa in 2010: A comparative risk assessment analysis. BMJ Open. 2015;

18. GBD 2015 Obesity Collaborators, Afshin A, Forouzanfar MH, Reitsma MB, Sur P, Estep K, et al. Health Effects of Overweight and Obesity in 195 Countries over 25 Years. N Engl J Med. 2017;

19. Danaei G, Singh GM, Paciorek CJ, Lin JK, Cowan MJ, Finucane MM, et al. The global cardiovascular risk transition: Associations of four metabolic risk factors with national income, urbanization, and western diet in 1980 and 2008. Circulation. 2013;

20. Sepanlou SG, Kamangar F, Poustchi H, Malekzadeh R. Reducing the burden of chronic diseases: a neglected agenda in Iranian health care system, requiring a plan for action. Arch Iran Med. 2010;

21. Sepanlou SG, Poustchi H, Kamangar F, Malekzadeh R. Effectiveness and feasibility of lifestyle and low-cost pharmacologic interventions in the prevention of chronic diseases: a review. Arch Iran Med. 2011;

22. Peykari N, Hashemi H, Dinarvand R, Haji-Aghajani M, Malekzadeh R, Sadrolsadat A, et al. National action plan for non-communicable diseases prevention and control in Iran; a response to emerging epidemic. Journal of Diabetes and Metabolic Disorders. 2017.

\section{Figures}




\section{National age-standardized}
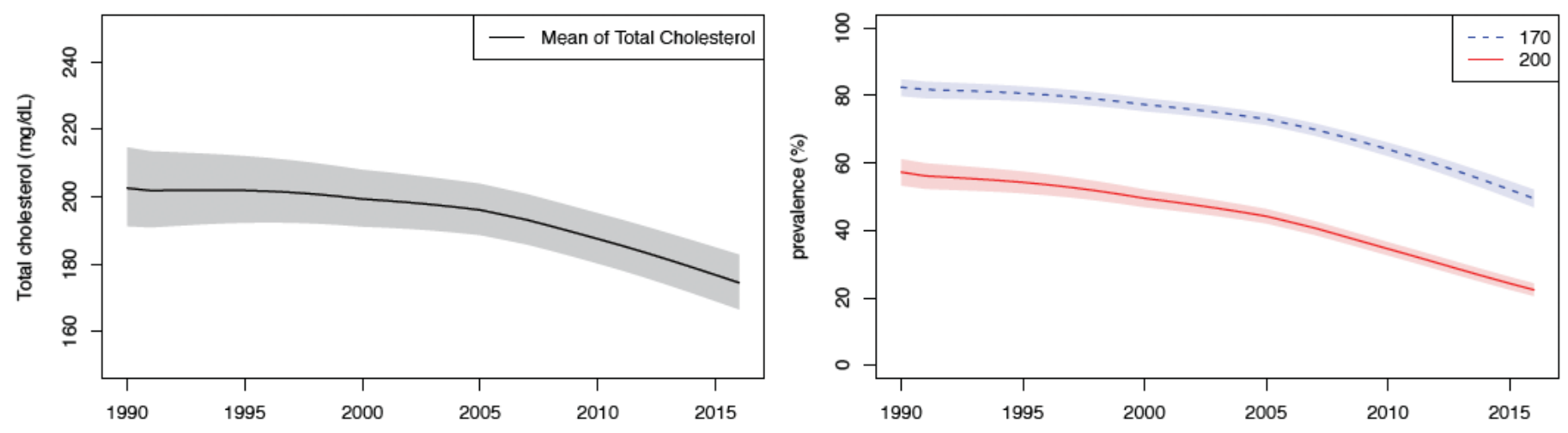

female
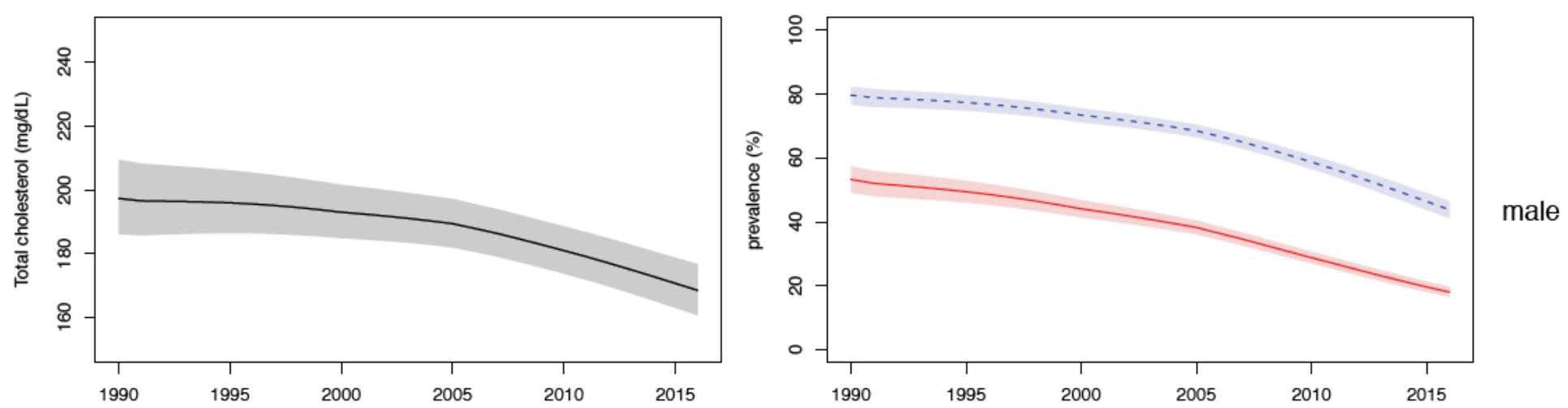

Figure 1

(linear trends): Trends in age-standardized mean total cholesterol and prevalence of elevated and raised total cholesterol by sex in people aged 25 years and older at national level from 1990 to 2016 . The lines show the posterior mean estimates and the shaded areas show the $95 \% \mathrm{Cl}$. See appendix for trends by province. 

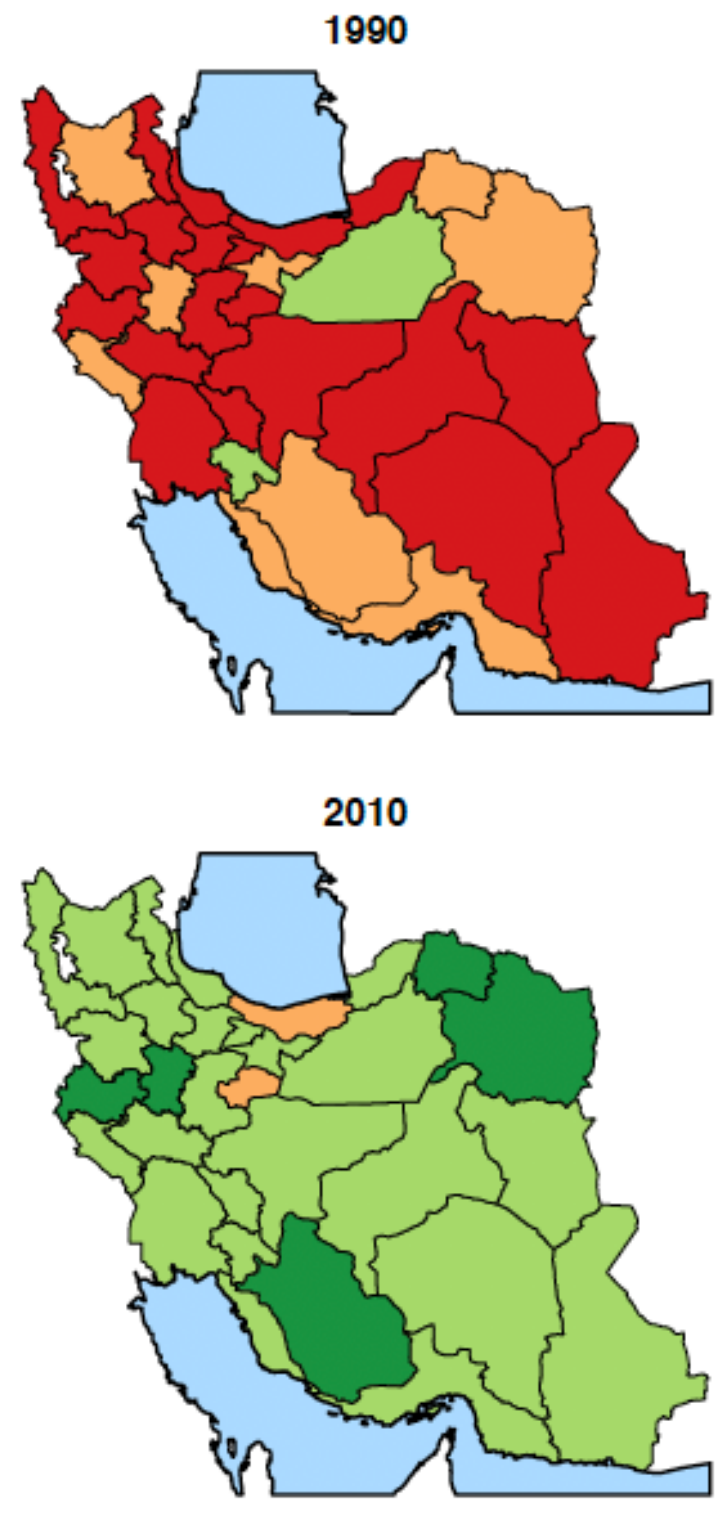
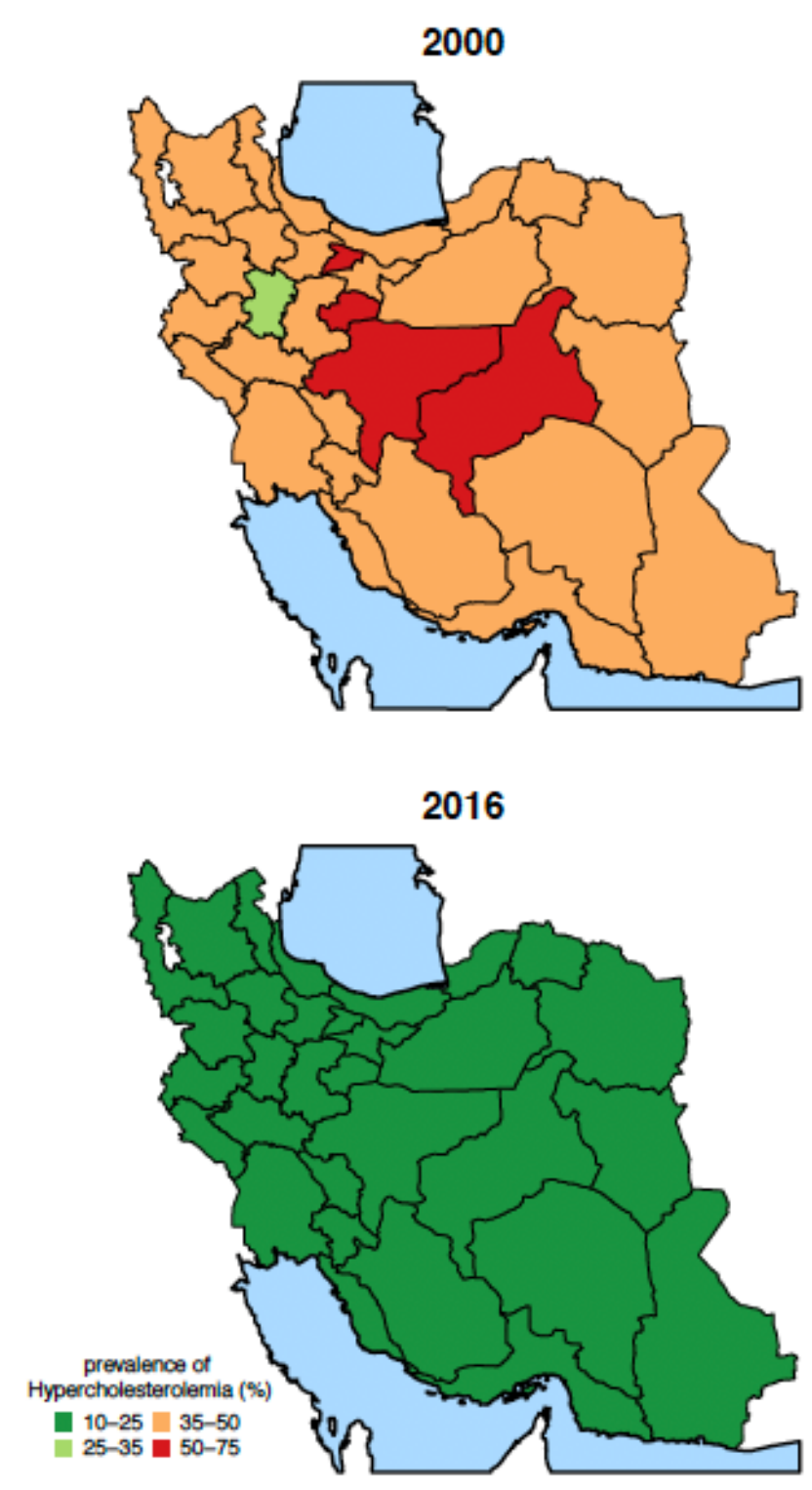

Figure 2

(map): Age-standardized prevalence of raised total cholesterol by sex and province in people aged 25 years and older in 1990, 2000, 2010, and 2016. 

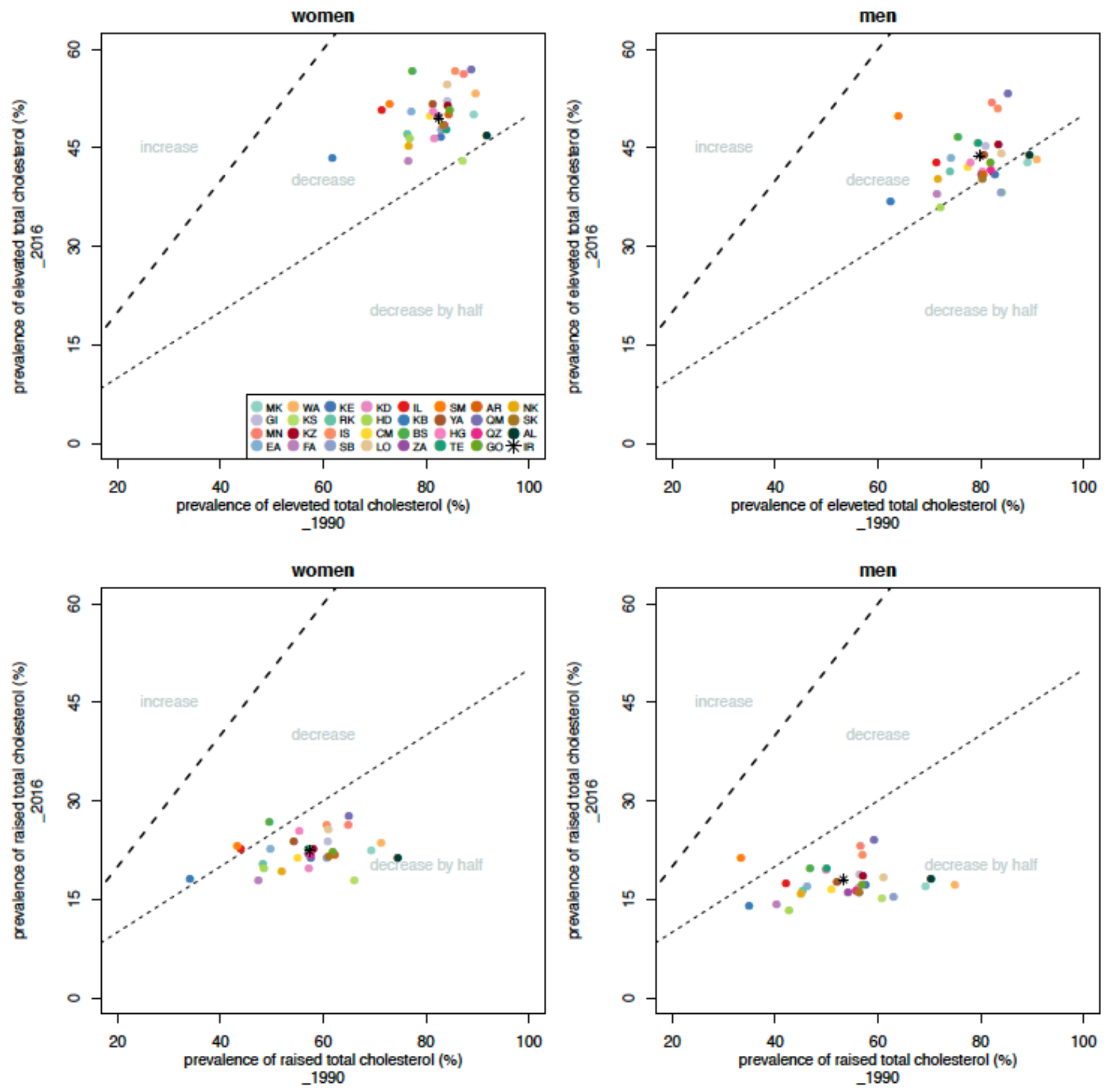

Figure 3

(Scatter Plot): Comparison of age-standardized prevalence of elevated and raised total cholesterol in adults by sex, by province between 1990 and 2016 


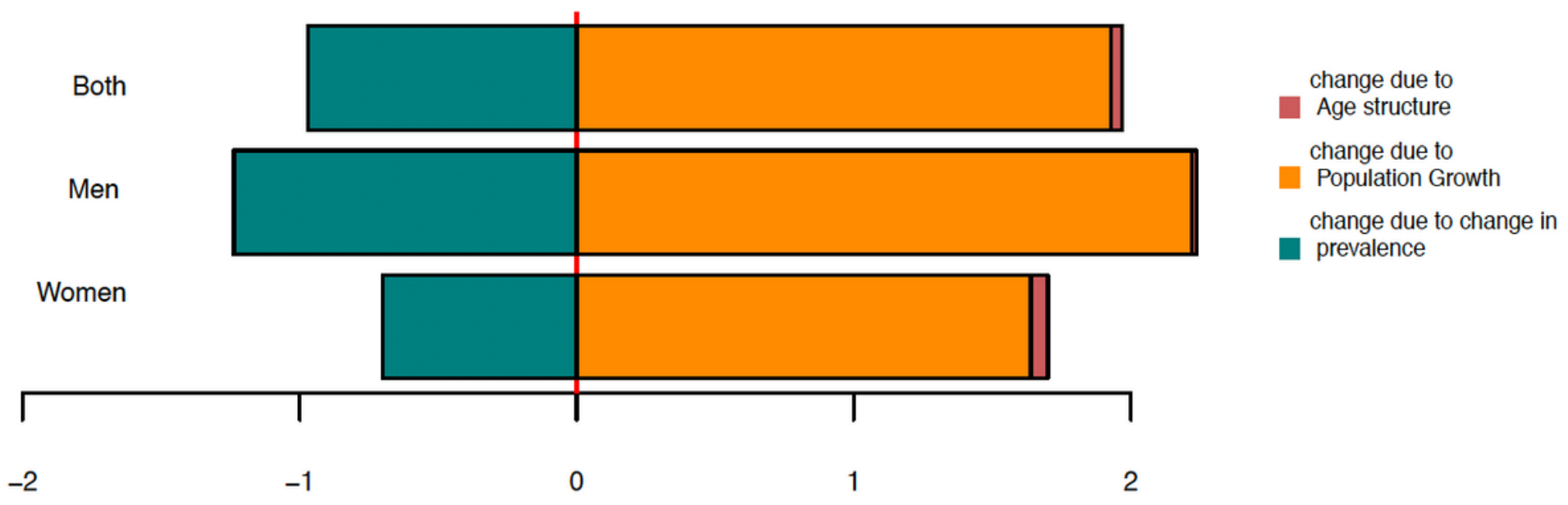

Figure 4

(histogram): shift in the distribution of the men total cholesterol from 1990 to 2016.

\section{Supplementary Files}

This is a list of supplementary files associated with this preprint. Click to download.

- Appendix.pdf 